\title{
EFECTOS DEL TIPO Y CONTENIDO DE LAS DESCRIPCIONES PRE-CONTACTO SOBRE LA CONDUCTA DE DISCRIMINACIÓN CONDICIONAL Y LAS DESCRIPCIONES POST-CONTACTO ${ }^{1}$
}

\author{
VÍCTOR GONZÁLEZ-BECERRA* \\ POSGRADO EN CIENCIA DEL COMPORTAMIENTO - OPCIÓN ANÁLISIS DE LA CONDUCTA \\ UNIVERSIDAD DE GUADALAJARA - MÉXICO \\ GERARDO ORTIZ \\ CENTRO DE ESTUDIOS E INVESTIGACIONES EN COMPORTAMIENTO \\ UNIVERSIDAD DE GUADALAJARA - MÉXICO
}

Recibido, marzo 12/2013

Concepto evaluación, noviembre 4/2013

Aceptado, noviembre 20/2013
Referencia: González-Becerra, V. \& Ortiz, G. (2014). Efectos del tipo y contenido de las descripciones precontacto sobre la conducta de discriminación condicional y las descripciones post-contacto. Acta Colombiana de Psicología, 17(1), 11-23. doi: 10.14718/ ACP.2014.17.1.2

Resumen

\begin{abstract}
Mediante un diseño factorial 2X3 (tipo de descripción pre-contacto, Específicas-Pertinentes (EP) y Correctas-Incorrectas (CI) X contenido de descripción, referentes a instancias, modalidades y relaciones) se constituyeron seis grupos de cuatro participantes que se expusieron a una tarea de igualación a la muestra de primer orden. Los resultados mostraron que los participantes que recibieron descripciones pre-contacto EP-modalidad y CI-relación obtuvieron los porcentajes de aciertos más altos en el entrenamiento (cercanos al 100\%), pero el porcentaje de aciertos disminuyó progresivamente en las pruebas de transferencia. La precisión y pertinencia de las descripciones post-contacto cambió en función de la ejecución, siendo irrelevante la precisión de la descripción pre-contacto recibida. Los resultados se discuten con relación a otras investigaciones en las que se han manipulado el tipo y/o contenido de las descripciones pre-contacto.

Palabras clave: descripción pre-contacto, descripción post-contacto, contenido, pruebas de transferencia, igualación de la muestra, teoría interconductual.
\end{abstract}

\section{EFFECTS OF TYPE AND CONTENT OF PRE-CONTACT DESCRIPTIONS ON CONDITIONAL DISCRIMINATION BEHAVIOR AND POST-CONTACT DESCRIPTIONS}

\begin{abstract}
With the use of a $3 \times 2$ factorial design (type of pre-contact description, Specific-Pertinent (SP), Correct-Incorrect (CI) X content concerning instances, modalities and relations) six groups of four participants were set up and exposed to a firstorder matching-to-sample task. Results showed that the participants who received pre-contact descriptions about SP-modality and CI-relation obtained the highest percentages of correct answers during training (close to 100\%), but the percentage of correct answers decreased progressively in the transfer tests. The accuracy and relevance of post-contact descriptions changed depending on implementation, whereas the accuracy of pre-contact description received was irrelevant. Results are discussed in relation to other research that has manipulated the type and / or content of the pre-contact descriptions.

Key Words: pre-contact description, post-contact description, content, transfer test, matching-to-sample, interbehavioral theory.
\end{abstract}

\footnotetext{
* Centro de Estudios e Investigaciones en Comportamiento, calle Francisco de Quevedo 180, colonia Arcos Vallarta. C. P. 44130. Guadalajara, Jalisco, México. victor.hugo.glez@gmail.com y oruga@cencar.udg.mx

1 El presente trabajo se presentó en el XX Congreso Mexicano de Análisis de la Conducta celebrado en Oaxtepec, Morelos, México en Agosto del 2010. Los autores contribuyeron en la misma proporción para la realización del estudio. El primer autor agradece al CONACYT por la beca \#2330889, la cual permitió su contribución del escrito.
} 


\title{
EFEITOS DO TIPO E CONTEÚDO DAS DESCRIÇÓES PRÉ-CONTATO SOBRE A CONDUTA DE DISCRIMINAÇÂO CONDICIONAL E AS DESCRIÇÓES PÓS-CONTATO
}

\author{
Resumo
}

\begin{abstract}
Mediante um desenho fatorial 2X3 (tipo de descrição pré-contato, Específicas-Pertinentes (EP) e Corretas-Incorretas (CI) X conteúdo de descrição, referentes a instâncias, modalidades e relações) constituíram-se seis grupos de quatro participantes que se expuseram a uma tarefa bde igualação à mostra de primeira ordem. Os resultados mostraram que os participantes que receberam descrições pré-contato EP-modalidade e CI-relação obtiveram as porcentagens de acerto mais altas no treinamento (próximos a 100\%), mas a porcentagem de acertos diminuiu progressivamente nos testes de transferência. A precisão e pertinência das descrições pós-contato mudou em função da execução, sendo irrelevante a precisão da descrição pré-contato recebida. Os resultados se discutem com relação a outras pesquisas nas quais manipularam-se o tipo e/ou conteúdo das descrições pré-contato.

Palavras chave: descrição, pré-contato, descrição pós-contato, conteúdo, testes de transferência, igualação da mostra, teoria, inter-condutual.
\end{abstract}

La Teoría Interconductual Ribesiana (TIR) (e.g.,Ribes, 2007; Ribes, 2004; Ribes, 1990; Ribes y López, 1985) se ha utilizado para estudiar el nivel de complejidad de la conducta de los organismos/individuos con base en las relaciones de interdependencia que se establecen en la interacción y no con base en la morfología de la conducta. Por ejemplo, la conducta verbal ecoica (tal como la definió Skinner, 1957) "galleta", puede emitirse morfológicamente tanto por un humano como por un loro, pero la conducta de ambos se puede diferenciar tomando en cuenta la estructura del sistema de contingencias (relaciones de interdependencia entre estímulos y respuestas) que se forma en cada interacción. En la TIR, a las estructuras contingenciales se les denomina funciones psicológicas y se clasifican, de acuerdo a su complejidad ascendente, como: contextuales, suplementarias, selectoras, sustitutivas referenciales y sustitutivas no referenciales. La complejidad de cada una de las funciones psicológicas se identifica por el mediador, el criterio de ajuste y el nivel de desligamiento funcional. El mediador es el factor crítico en la estructuración del campo de contingencias de la función psicológica. El criterio de ajuste hace referencia al requerimiento conductual (con relación a su función, no a su morfología) que un organismo debe desplegar en relación con una circunstancia contingencial específica. Por último, el desligamiento funcional hace referencia a la autonomía de respuesta del organismo con respecto a las propiedades de los estímulos (convencionales, ecológicas y fisicoquímicas) y a los parámetros espacio-temporales de los eventos con los que interactúa (e.g., Ribes, 2004; Ribes, 2007).

De acuerdo con el nivel de desligamiento, las tres primeras funciones psicológicas son intrasituacionales porque la conducta del organismo está ligada a una situación ${ }^{2}$, es decir, sólo se vinculan a una circunstancia y sólo tienen sentido en dicha circunstancia. La función sustitutiva referencial se considera extrasituacional porque la estructura contingencial de la conducta del individuo tiene sentido en dos situaciones, por lo cual la conducta se desliga de una situación para ligarse al sistema de contingencias de una situación diferente respecto de un mismo referente. Finalmente, la función sustitutiva no referencial se caracteriza por la mediación de relaciones referenciales por una respuesta convencional, al margen de toda contingencia situacional; en este sentido, dicho tipo de comportamiento se considera transituacional. A grandes rasgos, las conducta sustitutiva referencial se identifica por la capacidad de "ver" un objeto de dos formas (e.g., Ribes, 2011) con base en "la lógica" de dos situaciones, mientras que la conducta sustitutiva no referencial se caracteriza por la capacidad de crear una nueva situación a partir de la relación de dos o más situaciones (e.g., Torres, Ortiz, Rangel \& González, 2012).

De las funciones psicológicas anteriormente mencionadas la que más se ha estudiado ha sido la función sustitutiva referencial, la cual se ha evaluado generalmente mediante la tarea de igualación de la muestra (TIM). A los estudios que utilizan dicho procedimiento para evaluar el comportamiento

2 En este documento el término situación no hace referencia ni al lugar, ni a los objetos presentes en la interacción. La situación se entiende como el sistema de relaciones contingenciales entre eventos de estímulo y respuesta con diversas propiedades (i.e., fisicoquímicas, ecológicas, convencionales) que delimita la conducta de los organismos (e.g., Torres, Ortiz, Rangel \& González, 2012). Por lo tanto, la situación a la que se hace referencia es psicológica (e.g., Ribes, 1992) 
sustitutivo referencial se les ha denominado "estudios de lógica estándar" (Peña-Correal, Ordóñez, Fonseca \& Fonseca, 2012). Básicamente se asume en esta investigación que la conducta sustitutiva referencial se expresa cuando un participante pasa de responder a los eventos en una situación (por ejemplo, de una situación geométrica, relacionando figuras de acuerdo a su color o forma) con un criterio de relación (i.e., semejanza, diferencia, identidad) que tiene sentido con los eventos de otra situación (por ejemplo, de una situación aritmética, respondiendo a las unidades o decenas). Para evaluar dicha capacidad reactiva con la TIM se han diseñado pruebas de transferencia extradimensional en las que se presentan estímulos vinculados a situaciones diferentes de las del entrenamiento. Cuando el participante responde acertadamente tanto en el entrenamiento como en la prueba de transferencia extradimensional se asume que el comportamiento es sustitutivo referencial.

En otras investigaciones se ha asumido que en las pruebas de transferencia se puede evaluar comportamiento intrasituacional (selector), extrasituacional (sustitutivo referencial) y transituacional (sustitutivo no referencial) (e.g., Ribes, Ontiveros, Carvajal, Martínez \& Vargas, 2005; Ribes \& Serrano, 2006), tal como lo propuso Ribes (1990). En este sentido se ha asumido que las descripciones que se refieren a las propiedades instanciales (i.e., triángulo rojo con círculo rojo), modales (i.e., selecciona con el mismo color pero con otra forma) y relacionales (i.e., selecciona el diferente) de los estímulos tienen funcionalidad intrasituacional, extrasituacional y transituacional, respectivamente (e.g., Carpio, Pacheco, Carranza, Flores \& Canales, 2003; Irigoyen, Carpio, Jiménez, Silva \& Acuña, 2002; Serrano, García \& López, 2006; Serrano, García \& López, 2008; Serrano, García \& López, 2010; Villanueva, Mateos \& Flores, 2008). Bajo esta lógica se espera que a mayor nivel de funcionalidad en las descripciones mejor desempeño en las pruebas de transferencia. Sin embargo, en algunos casos las ejecuciones de los participantes expuestos a dicho tipo de descripciones no han mostrado correspondencia con el tipo de complejidad conductual esperado (e.g., Irigoyen et al., 2002; Serrano, García \& López, 2010).

Específicamente, en la investigación de Irigoyen et al. (2002) se evaluó el efecto del contenido de las descripciones sobre el entrenamiento y transferencia de una conducta de discriminación condicional. Tres grupos de participantes recibieron descripciones en las que cada grupo difería del otro por el tipo de contenido de éstas (referente a instancias, modalidades y relaciones), mientras que un cuarto grupo no recibió descripciones, pero fue retroalimentado cuando respondía correcta o incorrectamente. El objetivo era evaluar el efecto de dichas variables sobre la adquisición y transferencia de la conducta de discriminación condicional.
Los autores reportaron un mejor desempeño, tanto en el entrenamiento como en las pruebas de transferencia, en los participantes que no recibieron descripciones (grupo de retroalimentación correcta-incorrecta). También se encontró que de los participantes que recibieron descripciones, el que mejor desempeño tuvo en el entrenamiento y en las pruebas de transferencia fue uno de los que recibió descripciones intrasituacionales (refiriendo instancias). Estos resultados parecen mostrar que las descripciones son facilitadoras y no posibilitadoras del comportamiento complejo (PeñaCorreal, Ordóñez, Fonseca \& Fonseca, 2012), pero sigue resaltándose el hecho de que en algunos casos el tipo de descripción recibida corresponde al tipo de ejecución en las pruebas de transferencia.

Una interpretación del porqué las descripciones que se refieren a relaciones facilitan el comportamiento sustitutivo referencial se debe a que las propiedades que refieren relaciones (i.e., semejanza, diferencia, identidad) pueden tener sentido en dos situaciones distintas (i.e., semejanza geométrica y semejanza aritmética). En cambio, las descripciones que se refieren a las propiedades modales o instanciales de los estímulos sólo tienen sentido en una situación, pero no en otra. Por ejemplo, decir que para acertar es necesario tomar en cuenta el color, no tiene sentido en una situación aritmética; o que para acertar es necesario tener en cuenta el número diez, no tiene sentido en una situación geométrica. Por lo tanto, se asume que las descripciones pre-contacto que describen relaciones facilitan la generalización de la conducta aprendida en el entrenamiento a la prueba de transferencia extradimensional.

Por otra parte, existen algunas propuestas en las que se asume que para facilitar la abstracción y generalización de una conducta es necesario el contacto con las contingencias relevantes e irrelevantes de una situación, es decir, que el participante interactúe con las consecuencias que se relacionan con su comportamiento correcto e incorrecto en una circunstancia determinada (e.g., Engelmann \& Carnine; 1990; Ribes, 2000). En este sentido, Ortiz \& González (2010b) diseñaron descripciones en las que se presentan ejemplos de respuesta correctos e incorrectos (descripciones CI) con la finalidad de evaluar sus efectos en el entrenamiento y en la transferencia de una conducta de discriminación condicional en una TIM. Los autores encontraron que las descripciones CI facilitan la ejecución en el entrenamiento, pero no en las pruebas de transferencia. Sin embargo, en un estudio posterior, González-Becerra y Ortiz encontraron que las descripciones CI también facilitan el ajuste en la prueba de transferencia extradimensional.

Con la finalidad de evaluar la precisión y pertinencia de las descripciones, Ortiz, González \& Rosas (2008) desarrollaron una taxonomía para el análisis de las descripciones. 
Los autores denominaron descripciones pre-contacto al tipo de enunciado que se presentaba al participante previo a su ejecución en una tarea experimental. Dichas descripciones pueden adquirir la función de instrucción sólo si la conducta de quien la recibe corresponde a la conducta descrita. Por otra parte, denominaron descripciones post-contacto a los enunciados reportados después de la ejecución, sin importar su correspondencia con las contingencias experimentadas. Las descripciones, independientemente de su carácter pre o post-contacto, se clasifican de acuerdo a su presencia, relevancia, pertinencia y precisión en relación a las contingencias involucradas en una situación, dando como resultado las siguientes categorías: ausentes (A), irrelevantes (I), genéricas no pertinentes (GNP), específicas no pertinentes (ENP), genéricas pertinentes (GP) y específicas pertinentes (EP). Estas categorías pueden utilizarse para calificar la descripción de las contingencias de la situación de estímulo (objetos de interacción directa o indirecta), la respuesta y/o las consecuencias implicadas en un tipo de interacción.

En investigaciones en las que se han utilizado descripciones pre-contacto específicas y pertinentes (EP) se ha encontrado que éstas promueven altos porcentajes de aciertos en el entrenamiento (e.g., González-Becerra \& Ortiz, en revisión; Ortiz, 2005; Ortiz \& Cruz-Alaniz; 2010; Ortiz \& González, 2010a; Ortiz, de la Rosa, Padilla, Pulido \& Vélez, 2008; Ortiz, González, Rosas \& Alcaraz, 2006). Además se ha encontrado falta de correspondencia entre el tipo de descripciones recibidas (pre-contacto) y el tipo de descripciones realizadas (post-contacto) con relación a su precisión y en algunas ocasiones a su pertinencia.

Con base en lo comentado hasta ahora, el objetivo del presente estudio fue la evaluación del efecto del contenido y tipo de descripción pre-contacto en la ejecución y en las descripciones post-contacto de estudiantes universitarios expuestos a una TIM de primer orden. Para ello se varió el contenido de las descripciones pre-contacto haciendo referencia a las propiedades instanciales, modales y relacionales de los estímulos. El contenido de las descripciones se combinó con descripciones específicas-pertinentes (EP) y con descripciones de ejemplos de respuestas correctas e incorrectas (CI), con el objetivo de evaluar los efectos de las propiedades referidas en un ejemplo de respuesta relevante y en varios ejemplos de respuesta relevantes e irrelevantes. Por último, se utilizó la retroalimentación acumulada con la finalidad de poder evaluar en la primera sesión sólo el efecto de las descripciones pre-contacto sobre las ejecuciones de los participantes, y posteriormente evaluar, en las sesiones subsecuentes, el efecto de la combinación de las descripciones pre-contacto y de la retroalimentación.

\section{MÉTODO}

\section{Participantes}

Participaron 24 estudiantes universitarios con edades entre los 18 a 23 años, quienes fueron contactados a través de sus profesores de distintas materias y recibieron créditos por su asistencia al estudio. Todos ellos carecían de experiencia en la tarea experimental.

\section{Aparatos y materiales}

Todas las sesiones experimentales se llevaron a cabo en cuatro cubículos ubicados dentro de las instalaciones del Centro de Estudios e Investigaciones en Comportamiento de la Universidad de Guadalajara. Los cubículos tenían iluminación artificial y sus dimensiones eran aproximadamente de 3 metros de largo por 3 metros de ancho; en cada uno de ellos había un mueble con dos sillas y una computadora personal con ambiente Windows XP. La programación de los estímulos, la aplicación de la tarea experimental y el registro de las respuestas de los participantes se llevaron a cabo mediante el programa Toolbook Instructor II.

\section{Diseño}

Se utilizó un diseño factorial 3 X 2 (contenido de descripción X tipo de descripción) con lo cual se formaron seis grupos de participantes. Las variables que se manipularon fueron la referencia de propiedades instanciales, modales y relacionales de los estímulos en las descripciones específicas y pertinentes (EP) y en las descripciones de respuestas correctas e incorrectas (CI). Los grupos que resultaron de dicha combinación fueron el Grupo EP-instancia (Grupo 1), el Grupo EP-modalidad (Grupo 2), el Grupo EP-relación (Grupo 3), el Grupo CI-instancia (Grupo 4), CI-modalidad (Grupo 5) y CI-relación (Grupo 6). Todos los grupos enfrentaron una sesión de preprueba, una de posprueba, así como sesiones de pruebas de transferencia extrainstancial, extramodal, extrarelacional y extradimensional (de acuerdo a la matriz de transferencias propuesta por Varela \& Quintana, 1995); mientras que en el entrenamiento podían enfrentar de tres o cuatro sesiones (véase Tabla 1).

\section{Tarea experimental}

Como tarea experimental se utilizó un procedimiento de igualación de la muestra de primer orden (TIM-1). Cada ensayo consistió en la presentación de un estímulo muestra (EM) colocado en el centro de la pantalla y tres estímulos comparativos (ECo's) alineados horizontalmente en la parte inferior de ésta. Cada arreglo de estímulos presentaba, con respecto al EM, un ECo idéntico, uno semejante y otro diferente. La tarea de cada participante consistió en elegir, entre los tres ECo's, aquel que guardaba la relación 
Tabla 1

Diseño Experimental. La palabra retroalimentación está abreviada en la tabla como "retro".

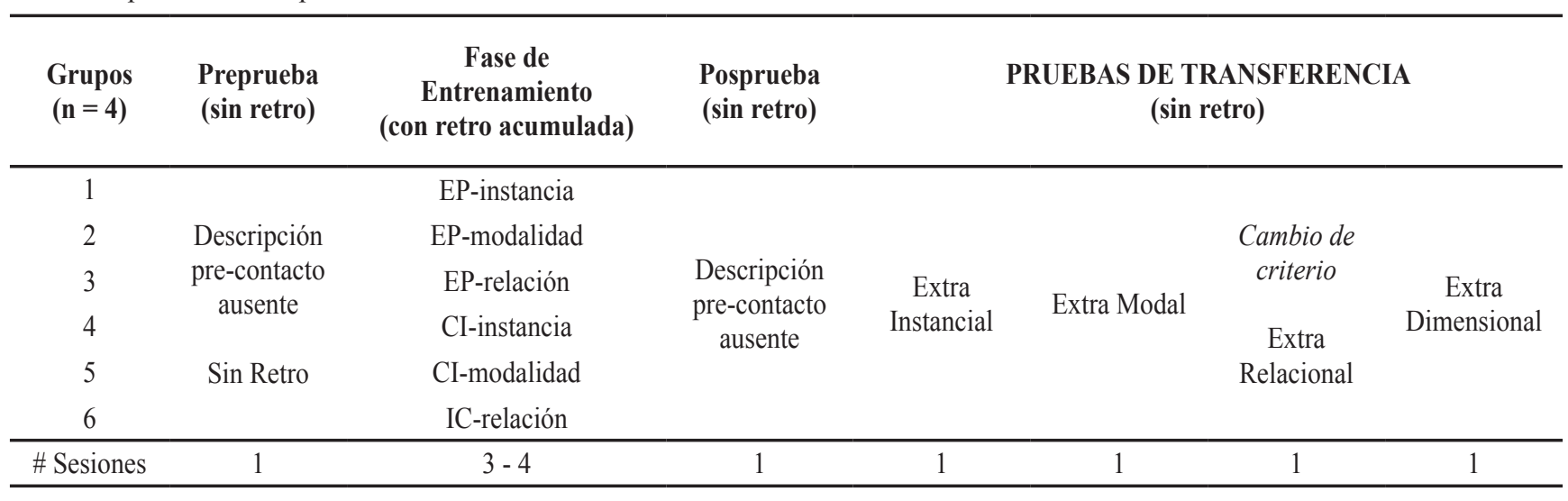

de semejanza con el EM, excepto en las pruebas de transferencia extrarelacional y extradimensional, en las cuales el criterio de relación cambiaba a identidad o diferencia.

Las figuras empleadas en las sesiones de preprueba, entrenamiento y posprueba fueron cuadros, triángulos, rectángulos y círculos de color blanco, rojo, verde y amarillo. En la prueba de transferencia extrainstancial se utilizaron pentágonos, hexágonos, rombos y trapecios de color naranja, azul, rosa y morado; en la prueba de transferencia extramodal se utilizaron las mismas figuras que en la prueba anterior, cambiando los tamaños de las figuras (chicas y grandes) y manteniendo un mismo color para todos los estímulos (gris). En la prueba de transferencia extrarelacional se utilizaron las mismas figuras que en la anterior prueba; sólo cambió el criterio de igualación a diferencia o identidad. Por último, en la transferencia extradimensional los estímulos fueron números de dos cifras, donde los Eco's se podían igualar indistintamente por decenas o unidades con respecto al EM; el criterio de igualación que se consideró correcto fue el de diferencia o identidad, dependiendo del criterio seleccionado por el participante en la prueba extrarelacional (véase Tabla 2). El arreglo y el tipo de estímulos utilizados en cada sesión fue el mismo para todos los grupos experimentales (véase Figura 1).

Tabla 2

Estímulos utilizados en las sesiones experimentales.

Sesión experimental
Preprueba, entrenamiento y
posprueba
$\begin{aligned} & \text { Prueba de transferencia } \\ & \text { extrainstancial } \\ & \text { modal } \\ & \text { Prueba de transferencia } \\ & \text { extradimensional }\end{aligned}$


Arreglo en preprueba, posprueba y entrenamiento

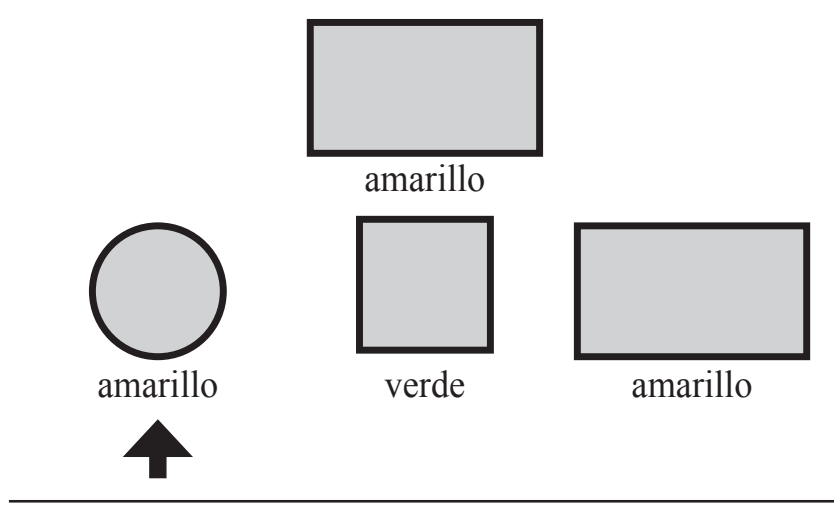

Arreglo en prueba de transferencia extrainstancial

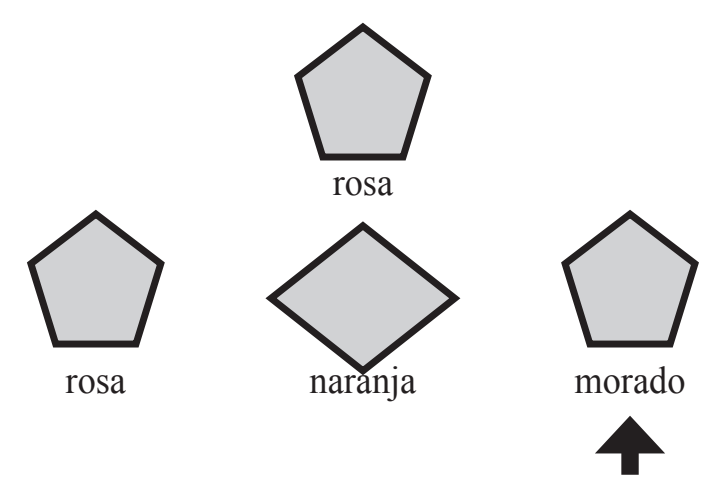

Arreglo en prueba de tranferencia extramodal
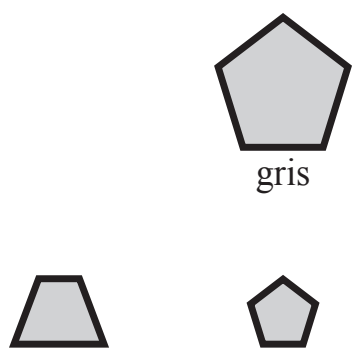

gris

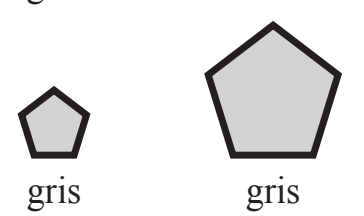

Arreglo en prueba de transferencia extradimensional

11

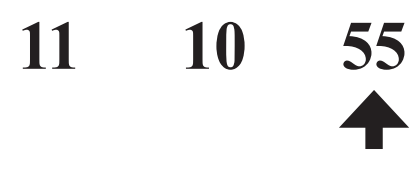

Figura 1. Arreglos de estímulos en las sesiones experimentales. En cada ejemplo de arreglo hay un estímulo comparativo diferente, uno idéntico y otro semejante. Las flechas indican el estímulo correcto en cada ejemplo (en todos los ejemplos que contienen figuras geométricas se señala la figura semejante, mientras que en los que contienen números se señala el diferente).

\section{Procedimiento}

$\mathrm{Al}$ inicio del estudio, a cada participante se le invitó a pasar a uno de los cubículos experimentales, se le pidió que se sentara frente al monitor y oralmente se le indicó que se trataba de un estudio sobre aprendizaje, agradeciéndosele su participación en el mismo. Además, se comentó a cada participante que no se trataba de una prueba proyectiva ni de inteligencia, que lo anterior era toda la información que el asistente podría brindarle y que al final del experimento se le explicarían los propósitos de dicho trabajo y los resultados de su ejecución, en caso de que así lo deseara.

Al comenzar cada sesión se presentó en la pantalla del monitor una diapositiva de bienvenida al experimento. En la siguiente diapositiva se encontraban descripciones que variaron dependiendo del grupo y de la fase experimental en la que se encontraba cada participante; también se presentó, en la parte superior de la diapositiva, un aviso de la sesión experimental enfrentada (i.e., preprueba, entrenamiento, posprueba y pruebas de transferencia). Posteriormente se presentaban, en una serie de diapositivas, los arreglos de estímulos a los que tenían que responder los participantes. En la penúltima diapositiva de la sesión se pedía al participante que describiera la forma en la que había resuelto la tarea y sólo en las sesiones de entrenamiento se indicaba la cantidad de respuestas correctas en la sesión. Al final de la sesión se presentaba en una diapositiva la información de que la sesión había terminado y que era necesario llamar al encargado del experimento. Cuando esto ocurría, el encargado guardaba los datos, cargaba en el computador una nueva sesión y dejaba al participante solo en el cubículo para que continuara con el experimento. La primera sesión experimental enfrentada por todos los participantes fue la de preprueba, en la cual no se retroalimentaba la ejecución. Posteriormente, en la fase de entrenamiento, la mitad de los participantes recibió, al final de cada sesión, retroalimentación sobre la cantidad de aciertos logrados (retroalimentación-acumulada), mientras que la otra mitad no recibió dicha retroalimentación (retroalimentación-ausente). Adicionalmente, la mitad recibió descripciones específicas y pertinentes (EP) de la situación de estímulo (SE) (i.e., 
los elementos del ambiente relevantes en la interacción), la respuesta efectiva en dicha situación de estímulo $(\mathrm{R}) \mathrm{y}$ las consecuencias derivadas de ello (C) (véase Ortiz, González \& Rosas, 2008); la otra mitad recibió descripciones a manera de ejemplos correctos e incorrectos (CI) basados en la propuesta de generalización del conocimiento de Engelmann \& Carnine (1991) (véase Anexo 1).

Todas las sesiones experimentales constaron de 36 ensayos y no tenían límite temporal de ejecución. La primera sesión enfrentada era la preprueba. Posteriormente, la fase de entrenamiento constaba de un mínimo de tres sesiones y un máximo de cuatro. Cuando los participantes lograban un porcentaje de aciertos mayor al $90 \%$ en tres sesiones consecutivas pasaban a la posprueba; de lo contrario, enfrentaban una cuarta sesión de entrenamiento. Las sesiones de preprueba y posprueba no eran retroalimentadas ni tenían descripciones. Por último, los participantes pasaban por una fase de pruebas de transferencia en las que no se retroalimentaba su ejecución. Al inicio de la prueba de transferencia extrarelacional se presentaba un aviso que decía: "Cambia el criterio de relación".

\section{RESULTADOS}

Los datos del presente experimento mostraron que los tipos de descripción, en combinación con el contenido de las descripciones, tuvieron efectos diferenciales en el desempeño de los participantes en el entrenamiento y en las pruebas de transferencia. Es de resaltar que aun cuando las descripciones que contenían relaciones auspiciaron altos porcentajes de acierto en el entrenamiento, éstas no lograron incidir de la misma manera en las pruebas de transferencia. Por otra parte, se encontraron descripciones genéricas pertinentes (GP) por parte de los participantes que tuvieron altos porcentajes de respuesta, independientemente del tipo o contenido de descripción recibida. Se procederá a describir los datos relacionados con las ejecuciones de los participantes y posteriormente sus descripciones.

En la figura 2 se muestra, en el eje de las ordenadas de cada gráfica, el porcentaje de aciertos obtenido por cada participante, y en el eje de las abscisas, las sesiones experimentales. Con rectángulos en color blanco se indica, de izquierda a derecha, el porcentaje de aciertos logrado en las sesiones de pre-prueba y post-prueba, respectivamente. Con triángulos en color blanco unidos por una línea negra se representó el porcentaje de aciertos alcanzado en cada una de las sesiones de entrenamiento. Por último, con barras en escala de grises se mostró el porcentaje de aciertos logrado en cada una de las sesiones de transferencia. Los gráficos de cada grupo de participantes se alinearon en orden ascendente (del Grupo 1 al Grupo 6) de manera vertical de izquierda a derecha, de tal forma que los tres grupos de participantes que recibieron descripciones pre-contacto EP quedaron del lado izquierdo de la figura 2, y del lado derecho, los que recibieron descripciones pre-contacto CI.

Con respecto a las ejecuciones en las sesiones de entrenamiento, se puede ver que los porcentajes de aciertos más bajos fueron obtenidos por los participantes que recibieron descripciones pre-contacto que contenían instancias (Grupo 1 y Grupo 4) y por los participantes que recibieron descripciones pre-contacto CI que contenían modalidades (Grupo 5). La excepción fueron los participantes P2, P13 y P15 (del Grupo 1 y el Grupo 4) que obtuvieron altos porcentajes de aciertos en las últimas sesiones de la fase de entrenamiento. En cuanto a los bajos porcentajes de aciertos mostrados por los participantes del Grupo 5 (CI-modalidad) se encuentra un efecto que contrasta con las ejecuciones de aquellos que recibieron descripciones pre-contacto con el mismo contenido (modalidad) pero de diferente tipo (EP) (Grupo 2), ya que estos últimos mostraron altos porcentajes de aciertos desde la primera hasta la última sesión de entrenamiento (excepto el participante P7). Por último, en cuanto a las ejecuciones en la fase de entrenamiento, se puede ver que los participantes del Grupo 6 (CI-relación) mostraron las mejores ejecuciones al obtener porcentajes de aciertos cercanos al 100\% desde la primera sesión, siendo el único grupo en el que sus cuatro participantes requirieron sólo de tres sesiones de entrenamiento, alcanzando, de manera consecutiva, porcentajes de aciertos superiores al $90 \%$. En contraste, los participantes del Grupo 3, que recibieron el mismo tipo de contenido (relación) en las descripciones pre-contacto de diferente tipo (EP), obtuvieron porcentajes de aciertos cercanos a cero en la primera sesión (a excepción del P10). Resalta que tres de los participantes del Grupo 3 (P10, P11 y P12) mejoraron su ejecución en las siguientes sesiones al aumentar el porcentaje de respuestas correctas casi al $100 \%$ en la última sesión (véase la figura 2).

Si se observa nuevamente la figura 2 se notará que los participantes que tuvieron altos porcentajes de aciertos en la última sesión de entrenamiento mantuvieron un porcentaje de aciertos similar en la posprueba y en la transferencia extrainstancial (T1). De los 17 participantes que mantuvieron un porcentaje de aciertos cercano al 100\% en T1, sólo 8 lograron un porcentaje de aciertos similar en la prueba de transferencia extramodal (T2) (P1, P2, P10, P11, P13, P15, $\mathrm{P} 22$ y P23). En la prueba de transferencia extrarelacional (T3) sólo cuatro participantes obtuvieron altos porcentajes de aciertos (P2, P10, P15 y P23); mientras que en la última prueba de transferencia (extradimensional-T4) sólo el participante P2 tuvo un desempeño efectivo. Observando los resultados encontrados en la fase de transferencia por cada 


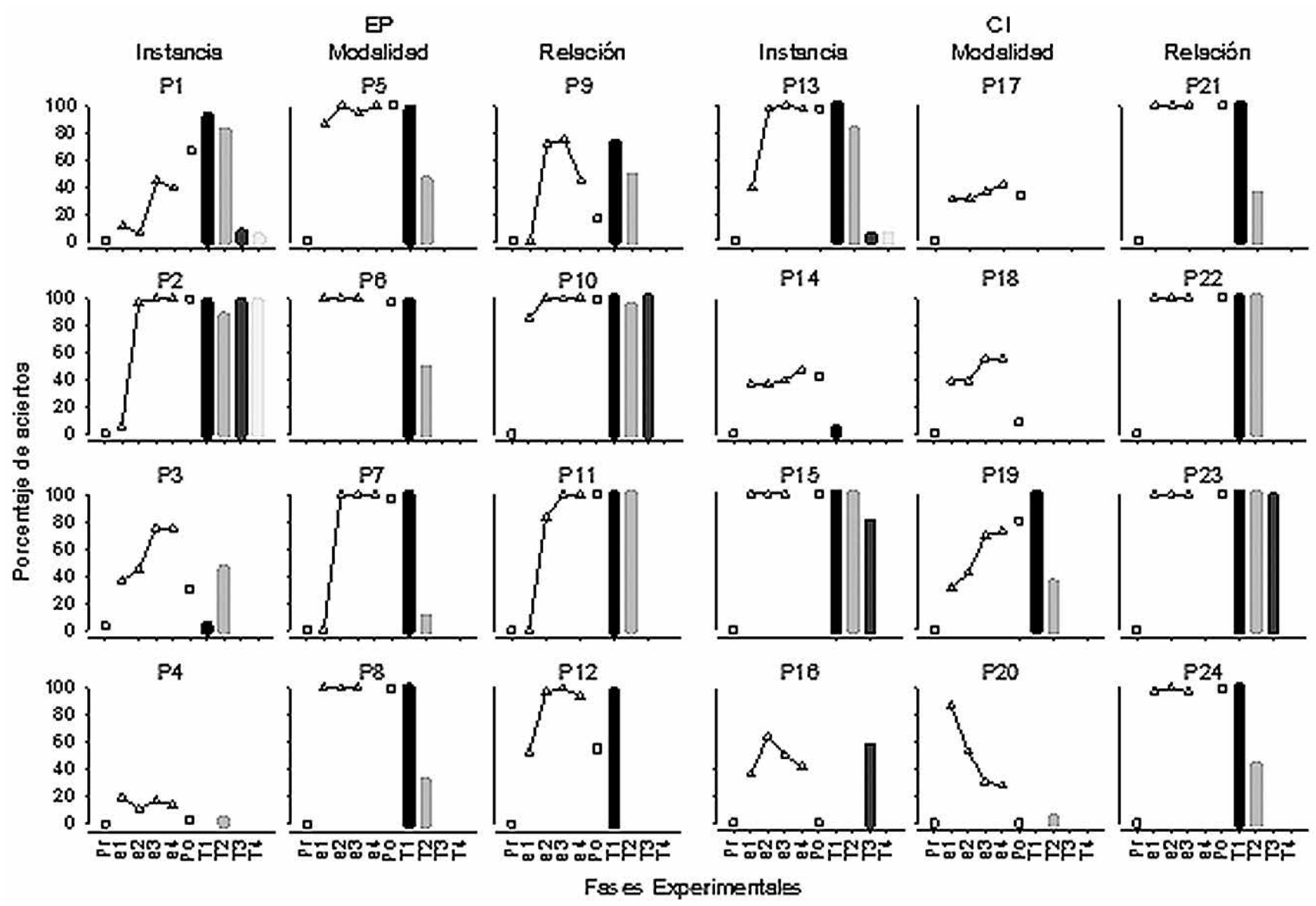

Figura 2. Porcentaje de aciertos obtenidos en cada sesión experimental por sujeto.

grupo, se destaca que el peor desempeño en dicha fase fue el de los participantes del Grupo 2 (EP-modalidad) y del Grupo 5 (CI-modalidad), y que los mejores resultados fueron obtenidos por un participante del Grupo 1 (EP-instancia), después de alcanzar porcentajes de aciertos cercanos al $100 \%$ en todas las pruebas de transferencia.

De las descripciones post-contacto de cada uno de los participantes sólo se analizó el componente Respuesta con base en la taxonomía propuesta por Ortiz, González \& Rosas (2008). Para que una descripción calificara como específica tenía que contener los siguientes elementos: a) el criterio de ajuste especificado para la situación, b) la acción principal solicitada para cumplir con el criterio de la tarea, y c) las maniobras o actividades requeridas para realizar dicha acción. Si la descripción contenía uno o dos de los anteriores elementos se consideraba genérica. La pertinencia o impertinencia de la descripción se relacionaba con la correspondencia o no correspondencia entre lo descrito y "lo ocurrido" contingencialmente; la irrelevancia se vinculaba a descripciones de elementos no pertenecientes a las contingencias de la tarea; y la ausencia, a la omisión de descripción. Con base en dichos criterios, en la figura 3 se presenta la precisión y pertinencia promedio de las descripciones post-contacto del componente Respuesta (R). En el grupo de gráficas de la izquierda se encuentran los gráficos concernientes al porcentaje de descripciones por tipo de cualidades reportadas en cada sesión de los grupos que recibieron descripciones EP (Grupos 1, 2 y 3), y en el grupo de gráficos de la derecha, los que recibieron descripciones CI (Grupos 4, 5 y 6). De manera horizontal, en tres filas compuestas por un par de grupos de gráficos se encuentran: en la parte superior, los grupos que recibieron descripciones con contenido de instancias; en la parte media, los grupos con descripciones que contenían modalidad; y en la parte inferior, los grupos con descripciones que contenían relación. En cada grupo de gráficos se alinearon en columnas aquellos concernientes a la cualidad de las descripciones post-contacto reportadas en promedio en los grupos experimentales en cada sesión experimental (a excepción de las sesiones de entrenamiento que quedaron representadas en un solo gráfico). De manera horizontal 
se ordenaron hileras, de abajo hacia arriba, del menor al mayor nivel de precisión y pertinencia descriptiva, según la taxonomía de Ortiz \& cols., (2008). Cada gráfico muestra con una barra en color gris el porcentaje de participantes que se reportó en cada tipo de descripción. Cuando la cantidad de participantes era de $0 \%$ se presentó una línea horizontal; cuando dicha cantidad aumentó en un $25 \%$, $50 \%, 75 \%$ y $100 \%$, en la misma proporción aumentó la altura de la barra. Cabe señalar que se representó en cada una de las sesiones experimentales el máximo valor cualitativo de las descripciones mostradas por los grupos; por lo tanto, dado que ninguna de las descripciones de los participantes se calificó como Específica Pertinente (EP), no apareció ninguna barra en dicha categoría.

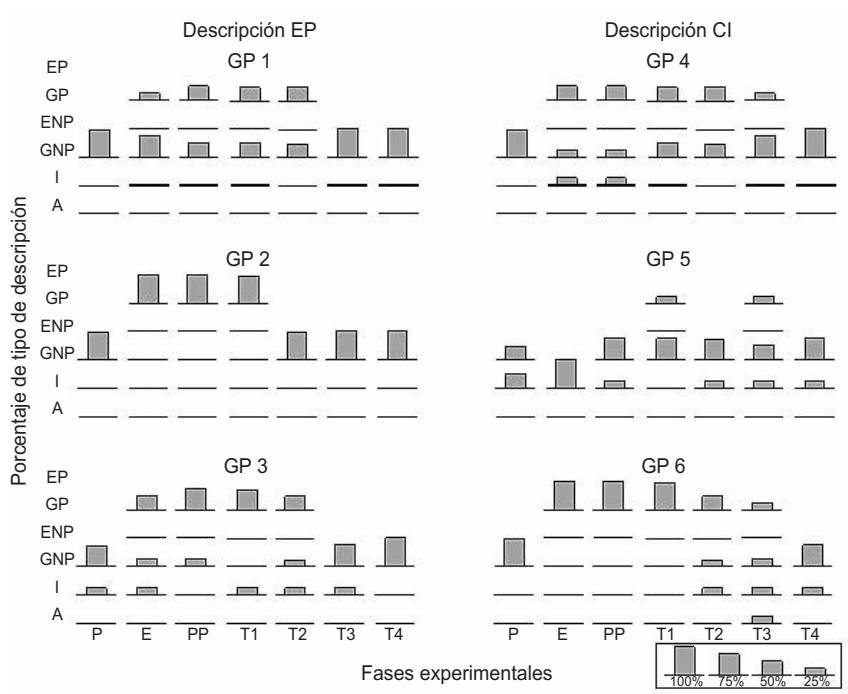

Figura 3. Nivel de descripción post-contacto por grupo en cada sesión experimental. En el recuadro de la esquina inferior derecha se encuentran los porcentajes que cada gráfico representó de acuerdo a su tamaño. Cada columna de gráficos fue señalada con la inicial del la fase experimental $(\mathrm{P}=$ pre-prueba, $\mathrm{E}=$ entrenamiento, $\mathrm{PP}=$ post-prueba, $\mathrm{T} 1=$ transferencia extrainstancial, $\mathrm{T} 2=$ transferencia extramodal, $\mathrm{T} 3=$ transferencia extrarelacional, $\mathrm{T} 4=$ transferencia extradimensional) y cada fila con las iniciales del tipo de descripción $(\mathrm{A}=$ ausente, $\mathrm{I}=$ irrelevante, $\mathrm{GNP}=$ genérico no pertinente, $\mathrm{ENP}=$ específico no pertinente, $\mathrm{GP}=$ genérico pertinente, $\mathrm{EP}=$ específico pertinente).

En la figura 3 se puede observar que la cualidad de las descripciones post-contacto reportadas por los participantes correspondió generalmente con el tipo de ejecución que éstos tuvieron en las fases experimentales (véase la figura 2). Por ejemplo, ningún participante obtuvo aciertos en la sesión de preprueba, y en correspondencia, sus descripciones fueron Genéricas-No Pertinentes (GNP). De manera similar, resalta que cuando los participantes de los Grupos 2 (EP-modalidad) y 6 (CI-relación) tuvieron un buen desempeño en la fase de entrenamiento, en la post-prueba y en la prueba de transferencia extrainstancial reportaron descripciones post-contacto genéricas pertinentes (GP) en las mismas sesiones. A grandes rasgos se puede ver que la pertinencia (respuestas acertadas) o falta de pertinencia (respuestas erróneas) de la ejecución de los participantes se vio correspondida en la mayoría de los casos por la pertinencia o falta de pertinencia de las descripciones post-contacto. Por una parte, se destaca que la precisión de la descripción post-contacto nunca fue específica, pese a que algunos participantes recibieron descripciones EP (Grupo 1, 2 y 3). También se destaca que las ejecuciones pertinentes de los participantes P2 y P10 en las pruebas de transferencia (véase la figura 2) no se ven reflejadas en descripciones post-contacto de la misma categoría en las gráficas del Grupo 1 y 3, respectivamente (véase la figura 3).

\section{DISCUSIÓN}

Aunque los participantes del Grupo 2 (EP-modalidad) y del Grupo 3 (CI-relación) mostraron altos porcentajes de aciertos en el entrenamiento, ninguno de ellos tuvo un buen desempeño en la prueba de transferencia extradimensional (T4). Este análisis va en contra de los estudios que reportan que las descripciones pre-contacto que refieren modalidades y relaciones mejoran el desempeño en las pruebas de transferencia (e.g., Carpio, Pacheco, Carranza, Flores \& Canales, 2003; González-Becerra, en revisión; Serrano, García \& López, 2006; Serrano, García \& López, 2008; Serrano, García \& López, 2010; Villanueva, Mateos \& Flores, 2008). Este resultado confirma que las descripciones sólo son facilitadoras y no posibilitadoras del comportamiento sustitutivo referencial (e.g., Peña-Correal et al., 2012). Aunque lo anterior es evidente, sigue siendo inquietante porqué en algunas ocasiones la referencia a propiedades modales y relacionales sí facilita el buen desempeño en las pruebas de transferencia y porqué en otras ocasiones no lo hace.

También se encontró que el único participante que obtuvo altos porcentajes de aciertos en T4, así como en las pruebas de transferencia precedentes, haya sido un participante que recibió descripciones en las que se referían a instancias (P2). A pesar de que en otras investigaciones se ha encontrado el mismo tipo de resultado en participantes que reciben descripciones referentes a instancias (e.g., Irigoyen et al., 2002; González-Becerra \& Ortiz, en revisión; Serrano et al., 2010) no se ha discutido por qué ocurre esto. El principal cuestionamiento a este tipo de resultados es: 
¿Cómo puede una descripción referente a las propiedades de una situación geométrica (triángulo rojo con cuadrado rojo) relacionarse con una situación aritmética en la que las propiedades en relación son unidades y decenas? González-Becerra y Ortiz (en revisión) argumentan que una posible explicación es que la aptitud sustitutiva referencial de vincular una conducta (selección del semejante, diferente e idéntico) en una situación geométrica y en una aritmética ya formaba parte del repertorio conductual de los participantes, previo a su participación en la investigación. Sin embargo, esa explicación no aclara porqué el resto de los participantes no mostró un comportamiento sustitutivo referencial a pesar de que ya formaba parte de su repertorio conductual.

Por otra parte, tanto en esta investigación como en otras en las que se ha manipulado el contenido de las descripciones, se ha encontrado que cuando se refieren instancias, la mayoría de los participantes que las reciben logran bajos porcentajes de aciertos en el entrenamiento y en las pruebas de transferencia (e.g., Serrano, García \& López, 2006; Serrano, García \& López, 2008; Villanueva, Mateos $\&$ Flores, 2008). Pero en esas mismas investigaciones se ha encontrado que los mejores resultados se encuentran en los participantes a los que se les refieren las propiedades modales y relacionales de los estímulos, lo cual no ocurrió de manera consistente en esta investigación. Específicamente, uno de los participantes del Grupo 3 (EP-relación) (P9) y todos los del Grupo 5 (CI-modalidad) (P13, P14, P15 y P16) tuvieron bajos porcentajes de aciertos en el entrenamiento y en las pruebas de transferencia.

De acuerdo con el supuesto de que la exposición a las contingencias relevantes e irrelevantes favorece la abstracción y generalización (e.g., Engelmann \& Canine, 1990; Ribes, 2000) se esperaba que las descripciones CI propiciaran altos porcentajes de aciertos en el entrenamiento y en las pruebas de transferencia. Sin embargo, lo que se encontró fue que ninguno de los participantes que recibió descripciones $\mathrm{CI}$ pudo mostrar un comportamiento sustitutivo referencial, incluso cuando algunas descripciones se referían a las propiedades relacionales de los estímulos. Estos resultados contrastan con los encontrados por González-Becerra \& Ortiz (en revisión) en un estudio en el que se dieron a los participantes descripciones CI, refiriendo instancias en una TIM de primer orden. Los autores encontraron que los participantes que recibieron descripciones CI obtuvieron porcentajes de aciertos cercanos al $100 \%$ en la fase de entrenamiento y en las pruebas de transferencia (incluso en la extradimensional). Por tal motivo se considera necesario realizar más investigaciones en las que quede claro porqué el mismo tipo de descripciones conlleva resultados diferentes.
Una posible explicación de la poca efectividad de los participantes para acertar en las pruebas de transferencia de esta investigación es que los resultados obtenidos dependieron, en cierta parte, del tipo de tarea utilizada (TIM de primer orden), pues en dicha tarea sólo se ha podido entrenar al participante en un criterio de relación (i.e., semejanza), ante los mismos objetos de estímulo (i.e., figuras geométricas). Este tipo de tarea pudo haber provocado que la conducta se ligara funcionalmente a las contingencias de la situación geométrica del entrenamiento. De hecho, en otras investigaciones similares en las que se utilizó la TIM de primer orden se ha encontrado que el desempeño de los participantes en las pruebas de transferencia no es efectivo (e.g., Ortiz \& González, 2010a; Ortiz \& González 2010b). Para seguir con el asunto de los resultados diferentes al mismo tipo de manipulaciones, en otras investigaciones en las que se ha utilizado una tarea TIM de primer orden se ha encontrado que los participantes pueden mostrar un buen desempeño en las pruebas de transferencia (e.g., González-Becerra \& Ortiz, en revisión; Serrano, García \& López, 2009). Sin embargo, sería bueno replicar esta investigación con una TIM de segundo orden porque los efectos de la combinación de las variables aquí manipuladas no se han evaluado con ese tipo de tarea.

En lo concerniente a las descripciones post-contacto reportadas por los participantes se encontró que generalmente correspondieron con la ejecución realizada, siendo pertinentes a las contingencias enfrentadas. Además, se encontró que no hubo correspondencia en cuanto a la precisión de la descripción post-contacto realizada y la descripción precontacto brindada, ya que los participantes que recibieron descripciones EP (Grupo 1, Grupo 2 y Grupo 3) reportaron descripciones post-contacto genéricas de igual forma que los participantes que recibieron las descripciones CI (Grupo 4, Grupo 5 y Grupo 6). Este tipo de inconsistencias entre la cualidad de las descripciones pre-contacto y post-contacto se ha encontrado en otras investigaciones (e.g., GonzálezBecerra \& Ortiz, en preparación; Ortiz, 2010; Ortiz \& CruzAlaniz, 2011; Ortiz \& González, 2010a; Ortiz \& González, 2010b; Ortiz, \& cols., 2006). Este efecto puede deberse a que la conducta pertinente y la descripción específica de la conducta pertinente no son comportamientos que se entrenen de manera simultánea solo por la exposición a descripciones específicas y a la retroalimentación. Por lo tanto, si se desea que las descripciones post-contacto del participante correspondan en cualidad y forma con las descripciones pre-precontacto recibidas, tal vez sea necesario entrenar previamente al participante en competencias descriptivas (e.g., Ortiz, 2005; Ortiz \& cols., 2006) si el objetivo de la investigación es la correspondencia entre descripciones pre y post-contacto. 
Aun cuando la taxonomía de Ortiz et al. (2008) permite clasificar las descripciones pre y post-contacto con base en su pertinencia y precisión (e.g., Ortiz, 2010; Ortiz \& CruzAlaniz, 2011; Ortiz \& González, 2010a; Ortiz \& González, 2010b; Ortiz, \& cols., 2006), dicha taxonomía no permite clasificar las propiedades de los eventos que se refieren (i.e., instancias, modalidades, relaciones). En este sentido, la taxonomía permitió identificar que el comportamiento efectivo (obtención de altos porcentajes de aciertos) de los participantes se relacionó, en la mayoría de los casos, con descripciones post-contacto genéricas y pertinentes. Pero la descripción post-contacto en el entrenamiento no se relacionó con la ejecución en las pruebas de transferencia, pues los participantes que ejecutaron efectivamente e hicieron descripciones post-contacto genéricas y pertinentes en el entrenamiento tuvieron desempeños diferentes en las pruebas de transferencia (véase la Figura 2). Específicamente se encontró que el único participante que mostró un comportamiento sustitutivo referencial (P2) describió su ejecución pertinente de la misma manera (genérica-pertinente) que los participantes que no respondieron sustitutivamente. Una estrategia para ampliar el análisis de las descripciones post-contacto sería evaluar la correspondencia entre las propiedades de los estímulos que refieren las descripciones pre-contacto y las que refieren los participantes en las descripciones post-contacto.

\section{REFERENCIAS}

Carpio, C., Pacheco, V., Carranza, N., Flores, C., \& Canales, C. (2003). Tipos de retroalimentación en el aprendizaje de términos metodológicos de la psicología experimental. Anales de Psicología, 19, 97-105.

Engelmann, S. \& Carnine, D. (1991). Theory of instruction: principles and applications. Eugene, RR. ADI Press.

González-Becerra, V., \& Ortiz, G. (en revisión). Efectos de la retroalimentación y el tipo de descripción sobre la conducta de discriminación condicional en una tarea de igualación de la muestra. Revista Mexicana de Análisis de la Conducta.

Irigoyen, J.J., Carpio, C., Jiménez, M., Silva, H., Acuña, K., \& Arroyo, A. (2002). Efecto de diferentes tipos funcionales de retroalimentación y su presentación parcial en el entrenamiento y transferencia de desempeños efectivos. Revista Sonorense de Psicología, 16, 23-31.

Ortiz, G. (2005). El papel de la retroalimentación y la precisión instruccional en la elaboración y uso de descripciones en tareas de discriminación condicional. Tesis de doctorado no publicada. Universidad de Guadalajara, Guadalajara.

Ortiz, G., \& Cruz-Alaniz, Y. (2011). El papel de la precisión instruccional y la retroalimentación en la ejecución y descripciones poscontacto. Revista Mexicana de Análisis de la Conducta, 37, 1, 69-87.
Ortiz, G., \& González (2010a). Efectos de precisión y pertinencia del componente situación de estímulo de una descripción precontacto. Revista Mexicana de Análisis de la Conducta, 26(1), 117-132.

Ortiz, G., \& González (2010b). Efecto de dos tipos de descripciones precontacto sobre la ejecución instrumental y descripciones poscontacto en tareas de igualación de la muestra. Acta Colombiana de Psicología, 13(1), 115-126.

Ortiz, G., de la Rosa, E., Padilla, R., Pulido, E. \& Vélez, H. (2008). Efectos de la precisión e historia instruccional en la insensibilidad a las contingencias en tarea de igualación de la muestra de primer orden con humanos. Acta comportamentalia, 16(2), 167-181.

Ortiz, G., González, A., \& Rosas, M. (2008). Una taxonomía para el análisis de descripciones pre y post contacto con arreglos contingenciales. Acta Colombiana de Psicología, $11(1), 45-53$.

Ortiz, G., González, A., Rosas, M., \& Alcaraz, F. (2006). Efectos de la precisión instruccional y la densidad de retroalimentación sobre el seguimiento instruccional, la elaboración y transmisión de descripciones en tareas de discriminación condicional. Acta Comportamentalia. 14, 103-130.

Peña-Correal, T., Ordóñez, S; Fonseca, J. \& Fonseca, L. (2012). La investigación empírica de la función sustitutiva referencial. En Padilla, A. \& Peréz-Almonacid, R. (Eds.) La función sustitutiva referencial: análisis histórico-crítico/avances y perspectivas (pp. 35-100). USA: University Press of the South.

Ribes, E. (2011). Perception and consciousness as behaviorreferred concepts. En Ribes, E. \& Burgos, J., Consciousness, Perception, and Behavior: Conceptual, Theoretical, and Methodological Issues (pp. 191-223). USA: University Press of the South

Ribes, E. (2007). Estados límites de campo, medios de contacto y análisis molar del comportamiento: reflexiones teóricas. Acta Comportamentalia, 15(2), 229-259.

Ribes, E. (2004). Acerca de las funciones psicológicas: un postscriptum. Acta comportamentalia, 12(2), 117-127.

Ribes, E. (2000). Instructions, rules, and abstraction: A misconstrued relation. Behavior and Philosophy, 28, 41-55.

Ribes, E. (1992). Sobre el tiempo y espacio psicológico. Acta Comportamentalia, 0, 71-84.

Ribes, E. (1990). Psicología General. México: Trillas.

Ribes, E., \& Serrano, M. (2006). Efectos de tres tipos de preentrenamiento en la adquisición y transferencia de una tarea de igualación de la muestra. Acta Comportamentalia, 14, 145-169.

Ribes, E., Ontiveros, C., Torres, C., Calderón, G, Carvajal, J., Martínez, C., \& Vargas, I. (2005). La igualación de la muestra como selección de los estímulos de segundo orden: efectos de dos procedimientos. Revista Mexicana de Análisis de la Conducta, 33, 1-22.

Ribes, E. \& López, F. (1985). Teoría de la conducta: un análisis de campo y paramétrico. México: Trillas. 
Serrano, G., García, G., \& López, A. (2010). Textos descriptivos de contingencia y retroalimentación en igualación de la muestra en humanos. International Journal of Psychology and Psychological Therapy, 10(1), 177-187.

Serrano, M., García, G., \& López, A. (2009). Efectos de la retroalimentación para las respuestas de igualación correctas $\mathrm{o}$ incorrectas en la adquisición y transferencia de discriminaciones condicionales. Revista Mexicana de Análisis de la Conducta, 35(1), 113-134.

Serrano, G., García, G., \& López, A. (2008). Textos descriptivos de contingencias como instrucciones iniciales en tareas de igualación de la muestra. Acta Comportamentalia, 16(3), 333-346.

Serrano, M., García, G., \& López, A. (2006). Textos descriptivos de contingencias como estímulos selectores en igualación de la muestra con humanos. Acta Comportamentalia, 14(2), 131-143.
Skinner, B. F. (1957). Verbal Behavior. USA: Prentice-Hall.

Torres, C., Ortiz, G., Rangel, N. \& González, V. (2012). Análisis del concepto de situación en la estructuración de las funciones psicológicas. En Padilla, M. \& Pérez-Almonacid, R. (Eds.) La función sustitutiva referencial: análisis histórico-crítico / avances y perspectivas (pp. 149-167). USA: University Press of the South.

Varela, J. \& Quintana, C. (1995). Comportamiento inteligente y su transferencia. Revista Mexicana de Análisis de la Conducta, 21(1), 47-66.

Villanueva, S., Mateos, R., \& Flores, C. (2008). Efectos del contenido y distribución de la retroalimentación sobre la discriminación condicional de segundo orden. Acta Comportamentalia, 16(2), 211-221. 


\section{ANEXO 1}

Descripciones EP y CI. En las descripciones $\mathrm{EP}$ las siglas $\mathrm{SE}=$ situación de estímulo, $\mathrm{R}=$ respuesta y $\mathrm{C}=$ consecuencias. Las descripciones variaban de acuerdo al contenido, el cual se indicaba con una "x", "y" y "z" para las descripciones EP; mientras que para las descripciones CI se indicaban con una "X", "Y" y "Z".

\section{CONTENIDO}

EP instancias $(\mathbf{x})$

EP modalidad (y)

EP relaciones $(\mathbf{z})$

\section{DESCRIPCION EP}

(SE) A continuación en la pantalla aparecerán cuatro figuras, una arriba y tres abajo, las cuales pueden ser cuadros, triángulos, círculos o rectángulos en rojo, verde, amarillo o blanco.

(R) Tu tarea consiste en elegir, de entre aquellas que pueden ser seleccionadas:

(x) la que creas que va con los siguientes ejemplos: cuando hubo un cuadro rojo en la parte de arriba el correcto fue un círculo rojo y cuando hubo un rectángulo verde el correcto fue un rectángulo amarillo.

(y) la figura que tenga el mismo color o forma, pero no ambas al mismo tiempo.

(z) la que tenga una relación de semejanza con la figura de arriba.

Para llevar a cabo tu elección deberás de mover y oprimir el botón izquierdo del "mouse".

(C) Al final de la sesión habrá una diapositiva en la que aparecerá en un recuadro el total de aciertos que obtuviste.

\section{CONTENIDO}

\section{DESCRIPCIONES CI}

Para realizar la siguiente tarea necesitas tomar en cuenta los siguientes ejemplos de elección correcta e incorrecta:

Ejemplos correctos $(X)$ :

Cuando hay un triángulo rojo arriba la elección correcta es el triángulo verde.

Cuando hay un triángulo amarillo arriba la elección correcta es el rectángulo amarillo.

Cuando hay un cuadrado rojo arriba la selección correcta es el cuadrado amarillo.

Cuando hay un cuadrado blanco arriba la selección correcta es el círculo blanco.

Ejemplos incorrectos $(X)$ :

Cuando hay un cuadrado rojo arriba la elección incorrecta es el cuadrado rojo.

Cuando hay un triángulo verde arriba la elección incorrecta es el triángulo verde.

Cuando hay un rectángulo blanco arriba la elección incorrecta es el a circulo amarillo.

Cuando hay un triángulo amarillo arriba la elección incorrecta es el cuadrado verde.

Ejemplos correctos (Y):

Cuando hay una figura triangular en color rojo arriba la elección correcta es la figura que tenga la misma forma pero no el mismo color.

Cuando hay una figura triangular color amarillo arriba la elección correcta es la figura que tenga el mismo color pero no la misma forma.

Cuando hay una figura rectangular en color rojo arriba la selección correcta es la figura que tenga la misma forma pero no el mismo color.

CI instancias (X) Cuando hay una figura rectangular en color blanco arriba la selección correcta es la figura que tenga el mismo color pero no la misma forma.

CI modalidad (Y) $\quad$ Ejemplos incorrectos $(Y)$ :

Cuando hay una figura circular en color rojo arriba la elección incorrecta es la figura que tenga el mismo color y la misma forma.

CI relaciones $(\mathbf{Z})$ forma.

Cuando hay un rectángulo blanco arriba la elección incorrecta es la figura que tenga el mismo color y la misma forma.

Cuando hay un triángulo amarillo arriba la elección incorrecta es la figura que no tenga ni el mismo color ni la misma forma.

\section{Ejemplos correctos (Z):}

Cuando hay un figura con forma triangular en coloro rojo arriba la elección correcta es la figura SEMEJANTE en color.

Cuando hay una figura con forma triangular en color amarillo arriba la elección correcta es la figura SEMEJANTE en forma.

Cuando hay una figura con forma cuadrada en color rojo arriba la selección correcta es la figura SEMEJANTE en color.

Cuando hay una figura con cuadrada en color blanco arriba la selección correcta es la figura SEMEJANTE en forma.

Ejemplos incorrectos $(Z)$ :

Cuando hay una figura triangular en color rojo arriba la elección incorrecta es la figura idéntica.

Cuando hay una figura triangular en color verde arriba la elección incorrecta es la figura diferente.

Cuando hay una figura rectangular en color blanco arriba la elección incorrecta es la figura idéntica.

Cuando hay una figura rectangular en color amarillo arriba la elección incorrecta es la figura diferente. 